\title{
PON-SC - program for identifying steric clashes caused by amino acid substitutions
}

\author{
Jelena Čalyševa ${ }^{1,2}$ and Mauno Vihinen ${ }^{1 *}$ (D)
}

\begin{abstract}
Background: Amino acid substitutions due to DNA nucleotide replacements are frequently disease-causing because of affecting functionally important sites. If the substituting amino acid does not fit into the protein, it causes structural alterations that are often harmful. Clashes of amino acids cause local or global structural changes. Testing structural compatibility of variations has been difficult due to the lack of a dedicated method that could handle vast amounts of variation data produced by next generation sequencing technologies.

Results: We developed a method, PON-SC, for detecting protein structural clashes due to amino acid substitutions. The method utilizes side chain rotamer library and tests whether any of the common rotamers can be fitted into the protein structure. The tool was tested both with variants that cause and do not cause clashes and found to have accuracy of 0.71 over five test datasets.
\end{abstract}

Conclusions: We developed a fast method for residue side chain clash detection. The method provides in addition to the prediction also visualization of the variant in three dimensional structure.

Keywords: Amino acid substitution, Variation interpretation, Structural clashes, Side chain rotamers

\section{Background}

Amino acid substitutions (AASs) are common variants and can have numerous effects and mechanisms [1]. A large number of prediction methods is available for investigating the tolerance of variants $[2-4]$ as well as their mechanisms including effects on protein stability [5-7], disorder [8], aggregation [9, 10], localization [11], interactions, electrostatics, RNA splicing [12, 13], tRNA molecules $[14,15]$ etc. $[16,17]$. Specific predictors are available for variants in some proteins including BRCA1 and 2 $[18,19]$, mismatch repair system proteins [20, 21], and Bruton tyrosine kinase (BTK) [22]. Recently it has become possible to predict also the phenotypic severity of diseaserelated variants [23].

Among the most common effects are structural alterations originating because the substituted residue cannot fit into the structure without causing (major) structural alterations. When the substituting residue does not fit in the structure, more or less drastic conformation change occurs as the consequence. Due to structural and physical

\footnotetext{
* Correspondence: mauno.vihinen@med.lu.se

${ }^{1}$ Protein Structure and Bioinformatics, Department of Experimental Medical

Science, Lund University, BMC B13, SE-22 184 Lund, Sweden

Full list of author information is available at the end of the article
}

reasons all side chain conformations are not possible or structurally favorable, instead there are certain most favored conformations called for rotamers. Structural alterations may occur due to several other reasons including new or deleted interactions such as salt bridges or disulfide bonds, altered ligand binding specificity and modified allosteric site.

Libraries of side chain rotamers have been determined either from crystal structures $[24,25]$ or based on molecular dynamics simulations [26]. These libraries contain residue rotamers independent of the backbone conformation or dependent on the local backbone, especially secondary structures. Methods have been described for side chain optimization $[27,28]$. These tools typically utilize a rotamer library, then apply an energy function to estimate rotamers and search algorithm to cover the three dimensional space.

Only a few tools have been developed for the prediction of the effect of AASs on protein structure [29-31]. These methods are either not available, do not have easy to use interface, or they are too slow to apply to large datasets, such as those generated by modern next generation sequencing (NGS) techniques. Methods for optimizing the side chain rotamers could be used for the task; however they are not designed to answer this question. To fill the 
gap, we developed a novel and fast method, PON-SC, to predict whether AASs are structurally compatible or if they form clashes. The method is applicable both for protein engineering applications when planning either stability increasing [32-34] or decreasing [35, 36] variations, as well as for the interpretation of variants [22, 37, 38]. If the introduced variant cannot be accommodated into the structure without severe clashes and consequent structural alterations, the variant is harmful, even diseasecausing. The performance of the method was benchmarked with known harmful and structurally compatible cases that were collected from several sources.

\section{Method}

A novel method was developed for side chain clash detection. The flowchart of the protocol is shown in Fig. 1. PON-SC analysis is based on fitting AASs to protein structures, thus three dimensional structure is needed. Even structural models can be used, but then it is up to the user to estimate how reliable the predictions are.

The method has decision points depending on the submission and prediction request (Fig. 1). The predictor was programmed with Python. Two approaches are used to make decisions about side chain compatibility; assumptions based on the location and type of the original and substituting residue as well as rotamer testing predictions.

\section{Processing of the input}

BioPython package [39] is used to parse the input file in PDB format. $\phi$ and $\psi$ torsion angles of amino acid backbones and accessibility of the side chains are calculated with STRIDE [40]. KDTree algorithm from scikit-learn package [41] is used to prepare the structures for rotamer

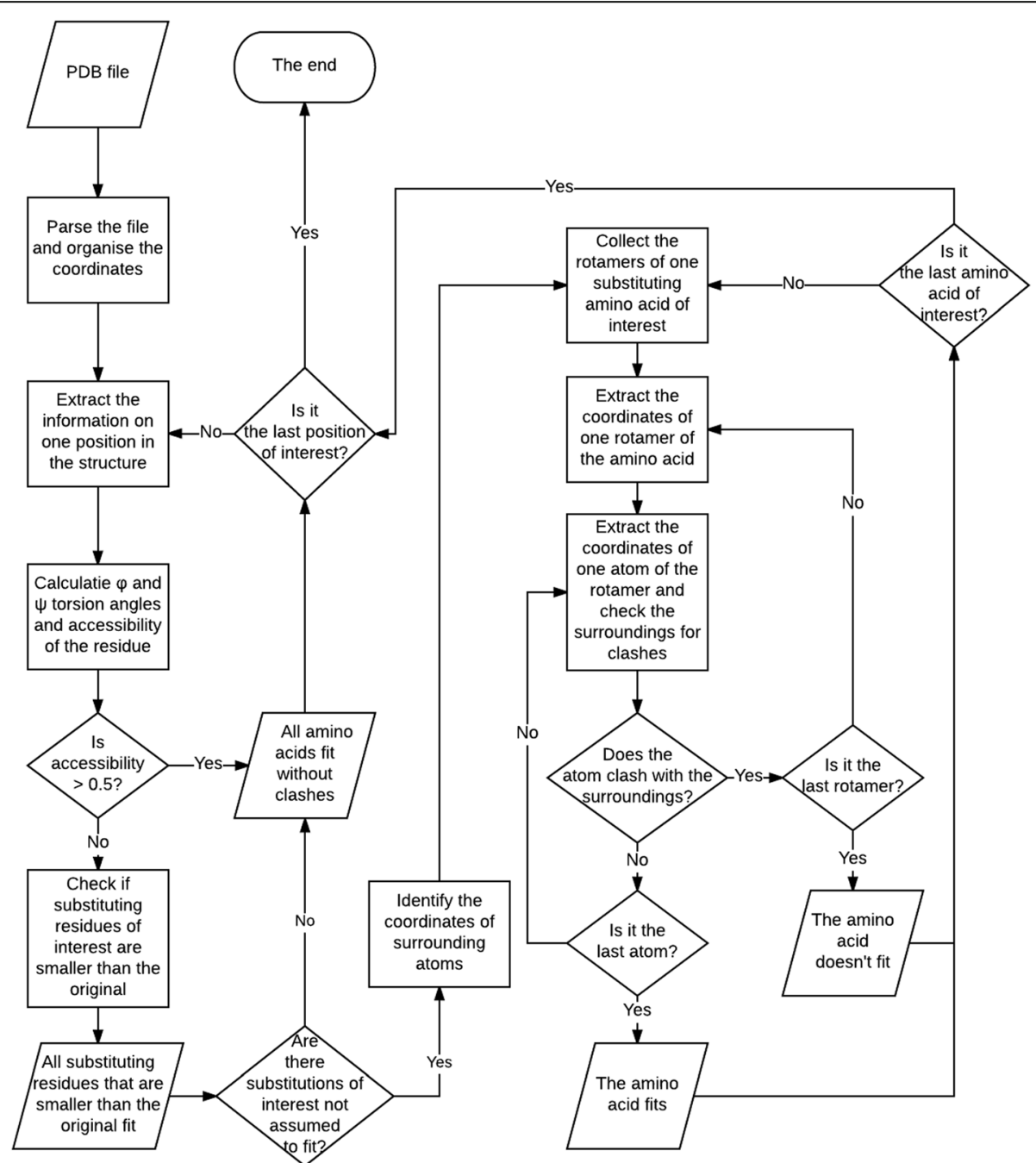

Fig. 1 The scheme of the method to identify amino acid substitutions causing clashes. Using PDB file as an input, the program iterates through all positions of interest in the structure, making assumptions and performing calculations for every substitution of interest, and providing information on whether the amino acid substitutions cause clashes in the structure or not 
tests. The amino acid side chain rotamers are added to the $\mathrm{C}_{\alpha}$ atom of the substituted residue.

\section{Assumptions about side chain alterations}

We use backbone-dependent rotamer library [24] for testing the space of potential side chain conformations. For $\phi$ and $\psi$ torsion angles of the selected residue, common rotamers for the substituting amino acid are considered.

To simplify and speed up the calculations, the following assumptions are made. First, if the ratio between the accessibility of the original residue and the highest possible accessibility of that residue type [42] is $\geq 0.5$ and the side chain is 3 or more heavy atoms long, all substitutions are assumed to fit into the structure. Thus, the method finds accessible positions that structurally allow all changes. Second, when the original amino acid is larger than the substituting one, no clashes are expected. Glycine is allowed in all positions, and smaller than original residues throughout the structure if they have a fitting structure. As an example, valine or leucine which have short but branched side chains are not directly assumed to be able to replace e.g. for arginine or lysine which have longer side chains. In these cases, the method tests whether the amino acid rotamers fit into the structure.

\section{Identifying fitting amino acids with calculations}

The furthermost possible clash is calculated to be in the distance to the $\mathrm{N}_{\mathrm{n}}$ atom of the straightest possible conformation of arginine and adding the van den Waals radius of nitrogen (1.64 $\AA$ ). Hydrogen atoms are ignored in the calculations. Variants left after the initial test are fitted in the available space around the residue. Side chain rotamers are tested to find one that fits into the structure. If the residue does not have any rotamer that would fit the substitution, it is considered to cause a clash and to be harmful (Fig. 2).

To calculate if a rotamer fits in the available space, rotation matrix for that rotamer is calculated and the clash detection is initiated. All atoms in the surroundings that possibly could form a clash are considered. For every atom starting after $C_{\beta}$ (atom1), the clash with every atom in the surrounding space (atom2) is calculated as follows:

$$
c=r_{\text {atom } 1}+r_{\text {atom } 2}-d_{\text {atoms }}-d_{\text {allowed }},
$$

where $c$ is the size of the overlap between the atoms, $r_{\text {atom }}$ is the van der Waals radius of the atom, $d_{\text {atoms }}$ is the distance between two atoms, and $d_{\text {allowed }}$ is the allowed clashing distance. The default $d_{\text {allowed }}$ value is $0.4 \AA$ [42]. The sum of the radii of atoms is set to $2.5 \AA$ when they form a hydrogen bond [43]. If both atoms are a part of cysteine side chain, the calculation is adjusted so that the minimal allowed distance between $\mathrm{C}_{\alpha}$ atoms

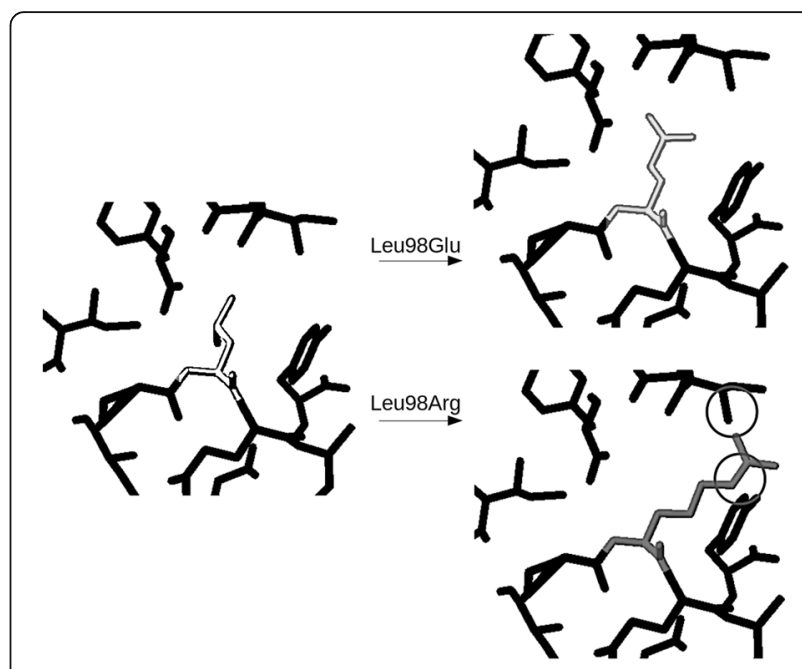

Fig. 2 An example of a clash between atoms caused by amino acid a substitution. Substitution of Leu98 (white) by Glu (top) in SH2D1A protein (PDB id 1D4W) causes no clashes with the surrounding residues, while substitution with Arg (bottom) causes clashes with lle84 and Tyr29 (indicated by circles)

is $4 \AA$ [44]. If the clash value is positive, the rotamer is discarded as not fitting and a new rotamer is taken until all of them have been tested or a fitting one is found.

\section{Datasets for validating the method}

The method was tested with structures from the PDB database [45]. First, PDB structure pairs differing by one amino acid were identified. After cleaning the data from incompatible PDB entries that either lacked information or when the positions in the structures did not match with the positions in corresponding protein sequences, the final set of 7795 variations was obtained. All the datasets used in this paper are available at VariBench database for variation prediction and testing database [46]. For comparison, clashing substitutions were identified by coupling SCWRL4 [27] and Probe [47] programs. SCWRL4 was used to build structures with the variant residues and Probe to detect clashes in them.

To further validate the method, several known cases of AASs having clashes were used. These included variants in CD40 ligand that is expressed in lymphocytes [37]. The structural effects of AASs were studied by bioinformatics methods in the structure of CD40LG tissue necrosis factor (TNF) homology domain (PDB ID 1ALY). 13 variations were reported to cause conformational damage and 19 not to affect the structure negatively.

Another dataset was for pathogenic Src homology 2 (SH2) domain variations in $12 \mathrm{SH} 2$ domains in 8 proteins [48]. The structures included the $\mathrm{SH} 2$ domain-containing 1A (SH2D1A), the zeta chain of $\mathrm{T}$ cell receptor associated protein kinase 70 (ZAP70) N-terminal SH2 domain, the phosphoinositide-3-kinase regulatory subunit 1 (PIK3R1) 
SH2 domain, the signal transducer and activator of transcription $1 \alpha$ (STAT1) SH2 domain, the BTK SH2 domain, and the RAS p21 protein activator 1 (RASA1) SH2 domain with corresponding PDB IDs 1D4W, 1M61, 2IUG, 1YVL, 2GE9 and 2GSB, respectively. Totally 28 structurally incompatible and 71 structurally compatible or neutral variations were obtained.

For human elastase, neutrophil expressed (ELANE, 1PPF) 23 AASs of which 3 were structurally compatible were obtained [38]. Variants in tumor protein p53 (TP53) [29] were included. There are 94 structures in the PDB database for the TP53 core domain/DNA complex, staphylococcal nuclease and the SH3 domain, PDB IDs 1TSR, 1STG and 1FMK, respectively. Totally 43 AASs cause clashes, while the number of amino acid substitutions not causing clashes is 121 .

Colorectal and breast cancer variations in TP53, KRAS proto-oncogene, GTPase (KRAS) and SMAD family member 4 (SMAD4) (1TSR, 1DD1 and 3GFT) [49] have been investigated at structural level. 10 out of the 31 studied substitutions were found to cause steric clashes.

All the datasets are available at VariBench at http:// structure.bmc.lu.se/VariBench/sidechain.php.

\section{Performance measures}

The method performance was assessed by using six performance scores [50] following guidelines for reporting [51]. When TP is the number of clash-causing variations predicted as not fitting into the structure, TN is the number of structure compatible variants that fit into the structure, FP is the number of fitting variations predicted as causing clash and FN is the number of clashing variations predicted as fitting into structure, the equations for computing the six performance measures are as follows:

$$
\text { Accuracy }=\frac{\mathrm{TP}+\mathrm{TN}}{\mathrm{TP}+\mathrm{TN}+\mathrm{FP}+\mathrm{FN}},
$$

Positive predictive value.

$$
P P V=\frac{\mathrm{TP}}{\mathrm{TP}+\mathrm{FP}},
$$

Negative predictive value

$$
N P V=\frac{\mathrm{TN}}{\mathrm{TN}+\mathrm{FN}}
$$

Sensitivity/True positive rate

$$
T P R=\frac{\mathrm{TP}}{\mathrm{TP}+\mathrm{FN}}
$$

Specificity/True negative rate

$$
T N R=\frac{\mathrm{TN}}{\mathrm{TN}+\mathrm{FP}}
$$

and Matthews Correlation Coefficient

$$
M C C=\frac{(\mathrm{TP} \times \mathrm{TN})-(\mathrm{FP} \times \mathrm{FN})}{\sqrt{(\mathrm{TP}+\mathrm{FN}) \times(\mathrm{TP}+\mathrm{FP}) \times(\mathrm{TN}+\mathrm{FN}) \times(\mathrm{TN}+\mathrm{FP})}},
$$

where NPV is negative predictive value and PPV is positive predictive value and MCC is Matthews correlation coefficient.

\section{Implementation}

The program has web interface that was programmed with Python using Django platform. There are several options for submitting variants. By providing PDB ID, the structure will be downloaded from PDB. Users need to note that PON-SC will consider clashes with all atoms in the PDB file. It may be necessary to exclude solvent atoms other than waters, which are automatically excluded from the calculations. It is possible to submit variants in several proteins at one time. Further, the user can choose to submit own PDB coordinates.

The variants to be analyzed are listed one per line. If only the position number is provided PON-SC predicts all 19 AASs in that position. The variant visualizations are available by JavaScript Protein Viewer (https:// biasmv.github.io/pv/). The results can be obtained while waiting or by e-mail. PON-SC is freely accessible at http://structure.bmc.lu.se/PON-SC.

\section{Results and discussion}

To identify AASs causing clashes in structures, various properties of the amino acids and polypeptides have to be considered. These include different radii of interacting atoms, bond lengths, hydrogen and disulfide bonds, the limited flexibility of the side chain in the structure, errors in resolved protein structures, etc. PON-SC considers clashes if the substituting residue comes too close to other atoms in the structure. The method considers clashes also with ligands and heteroatoms, if included to the structure. Waters are automatically removed from the calculations.

\section{Performance of the program}

PON-SC is very fast, it takes on average $0.05 \mathrm{~s}$ to evaluate a substitution once the PDB file is downloaded. SCWRL4 [27] is a widely used method for side chain rotamer optimization. It is used together with Probe [47], an atomic packing evaluation tool, to detect clashes. These programs are substantially slower than PON-SC because several intermediate steps are required e.g. to create new protein structures for every amino acid substitution and parsing the outputs of the programs. Calculation for a variant takes on average $1.3 \mathrm{~s}$ 
per substitution for SCWRL4 + Probe, i.e. it is 26 times slower than PON-SC. Note that SCWRL4 and Probe are not combined into a package, instead are run separately. SCWRL4 and PON-SC use the same rotamer library.

We tested the method performance with datasets of known cases. Data for clashes are limited as there are usually no structures for them. AASs that are clash-free were collected by identifying PDB structures that had only one residue difference. 7795 such cases were found and predicted both with PON-SC and SCWRL4 + Probe (Table 1). $77.4 \%$ of these AAS were predicted by PON-SC not to cause clashes. The performance of SCWRL4 + Probe is somewhat higher, having correct predictions in $83.6 \%$ of the cases. This test was made to address how many negative cases i.e. tolerated AASs are correctly predicted.

The reason for detecting clashes among these cases is at least partly due to structural rearrangements outside the variant position. Alterations due to AASs can appear in several amino acids $[29,52]$ not only in the substitution site. Neither PON-SC nor SCWRL + Probe combination can detect these. However, SCWRL4 + Probe performs better since SCWRL4 allows flexibility for the backbone and side chain as it is an optimization tool.

\section{Performance for different AASs}

Neither of the methods had problems fitting smaller amino acids in the available space in the structure (Table 1).
Substitutions to alanine or glycine did not cause clashes. Substitutions to cysteine and serine formed clashes only in a few cases. The reason behind SCWRL4 + Probe identifying clashes in the case of introducing cysteine could be that the method didn't account for disulfide bridges in the structure. PON-SC did not have any problems with substitutions to cysteine.

In the case of substitutions to larger amino acids, the situation is more variable. Some of the differences between the methods can be explained by the higher flexibility allowed by SCWRL4 including alterations to the polypeptide backbone. Proline is the most problematic side chain for PON-SC to predict. This is because the method provides freedom only for side chains, whereas in proline substitutions also the backbone is altered. Therefore, the method over-predicts clashes in proline substitutions.

In case of asparagine, aspartic acid and phenylalanine PON-SC identified far less clashes than SCWRL + Probe. Interestingly, the situation is the opposite for the related substitutions by glutamine and glutamate. In conclusion, the two approaches, PON-SC and SCWRL4+Probe, performed overall quite similarly; however, there were major substitution type-specific differences.

\section{Comparison to previous studies of steric clashes}

A real test for a predictor is to use both positive and negative cases. We collected five datasets from different

Table 1 Number of predicted clashes by amino acid types in PDB structures that tolerate substitutions

\begin{tabular}{|c|c|c|c|c|c|c|c|}
\hline & PON-SC number & PON-SC (\%) & SCWRL+ Probe number & SCWRL+ Probe (\%) & Both $^{a}$ number & Both (\%) & Total $^{b}$ \\
\hline Alanine & 0 & 0 & 0 & 0 & 0 & 0 & 1165 \\
\hline Arginine & 86 & 25.52 & 11 & 3.26 & 15 & 4.45 & 337 \\
\hline Asparagine & 37 & 8.22 & 112 & 24.89 & 7 & 1.56 & 450 \\
\hline Aspartic acid & 38 & 8.35 & 125 & 27.47 & 13 & 2.85 & 455 \\
\hline Cysteine & 0 & 0 & 4 & 1.316 & 0 & 0 & 304 \\
\hline Glutamic acid & 92 & 18.70 & 42 & 8.54 & 42 & 8.54 & 492 \\
\hline Glutamine & 42 & 15 & 31 & 11.07 & 22 & 7.86 & 280 \\
\hline Glycine & 0 & 0 & 0 & 0 & 0 & 0 & 393 \\
\hline Histidine & 74 & 21.70 & 45 & 13.20 & 38 & 11.14 & 341 \\
\hline Isoleucine & 94 & 33.45 & 38 & 13.52 & 42 & 14.95 & 281 \\
\hline Leucine & 96 & 25.26 & 39 & 10.26 & 27 & 7.11 & 380 \\
\hline Lysine & 53 & 19.41 & 6 & 2.20 & 5 & 1.83 & 273 \\
\hline Methionine & 93 & 32.63 & 11 & 3.86 & 10 & 3.51 & 285 \\
\hline Phenylalanine & 86 & 18.86 & 118 & 25.88 & 95 & 20.83 & 456 \\
\hline Proline & 90 & 76.92 & 0 & 0 & 0 & 0 & 117 \\
\hline Serine & 5 & 0.89 & 4 & 0.71 & 0 & 0 & 561 \\
\hline Threonine & 78 & 24.68 & 30 & 9.49 & 28 & 8.86 & 316 \\
\hline Tryptophan & 54 & 29.19 & 29 & 15.68 & 58 & 31.35 & 185 \\
\hline Tyrosine & 110 & 30.05 & 76 & 20.77 & 71 & 19.40 & 366 \\
\hline Valine & 122 & 34.08 & 41 & 11.45 & 42 & 11.73 & 358 \\
\hline
\end{tabular}

${ }^{\mathrm{a}}$ Does not include cases listed in PON-SC and SCWRL+Probe columns. ${ }^{\mathrm{b}}$ Total number of substitutions in the dataset. 
Table 2 Validation of the method performance

\begin{tabular}{llllllllllll}
\hline Study & TP & FP & TN & FN & Total & NPV & PPV & Sensitivity & Specificity & Accuracy & MCC \\
\hline CD40LG & 9 & 1 & 18 & 4 & 32 & 0.75 & 093 & 0.69 & 0.95 & 0.82 & 0.66 \\
SH2 & 13 & 25 & 46 & 15 & 99 & 0.54 & 0.57 & 0.46 & 0.65 & 0.55 & 0.11 \\
ELANE & 16 & 1 & 2 & 4 & 23 & 0.77 & 0.70 & 0.80 & 0.65 & 0.73 \\
TP53 & 27 & 19 & 102 & 16 & 164 & 0.69 & 0.80 & 0.63 & 0.84 & 0.74 & 0.48 \\
CANCER & 7 & 5 & 16 & 3 & 31 & 0.72 & 0.75 & 0.71 & 0.76 & 0.74 & 0.47 \\
Total/Average & 72 & 51 & 184 & 42 & 349 & 0.69 & 0.74 & 0.66 & 0.77 & 0.71 & 0.43 \\
\hline
\end{tabular}

studies. Since protein structures with major clashes cannot be investigated with e.g. crystallography and since negative results are not frequently published, there are not many cases with reported clashes in literature and databases. After extensive search we found five datasets that we used to test the performance of the tool.

The average performance over all the datasets is as follows: sensitivity is 0.66 , specificity 0.77 , accuracy 0.71 and MCC 0.43 (Table 2). Only the datasets for TP53 and cancers have specifically addressed the clashes of the substitutions. PON-SC has typically higher specificity than sensitivity, i.e. it predicts clashes with somewhat lower accuracy than tolerated variants. Exception is the ELANE dataset, but since this is a small set, minor random effects may have major impact. The average accuracy of 0.71 indicates that the method is rather reliable, and because of its speed, it can thus be used for analysis of even large datasets. The overall quality scores are more relevant since the individual datasets are quite small.

The PON-SC program does not give information on the severity of a clash, only that it occurs. The method is implemented such that the detected clashes are highly likely structurally incompatible and therefore harmful. For visualization of the results the PON-SC tool utilizes the JavaScript Protein Viewer plugin that shows the original and variant residues in three dimensional structures. The rotamer used for the visualization is not necessarily the best fitting one but it is the most common of the fitting ones, as the rotamers are tested in the decreasing order of frequency. To save time, the program ends the search after finding the first fitting rotamer and then that one is visualized. For the prediction purposes it is sufficient to find one rotamer that allows fitting the novel side chain.

For comparison, the results for the SCWRL + Probe are shown in Additional file 1: Table S1. On these datasets PON-SC has somewhat better performance and also displays more balanced results in regards to the measures. The MCC and accuracy are 0.29 and 0.43 , and 0.64 and 0.71 for SCWRL + Probe and PON-SC, respectively. PON-SC had equal or better values for all the five tested variation sets.

\section{Conclusions}

PON-SC is a novel method for varient effect prediction. It detects structural clashes due to AASs based on protein three dimensional strucutre, side chain rotamer library, structural assumptions and calculations. The method has a relatively high performance, accuracy being 0.71 over several datasets. PON-SC is currently the only tool that can be used for large scale analysis e.g. of NGS datasets. Side chain replacements can be visualized in protein structures.

\section{Availability and requirements}

Project name: PON-SC.

Project home page: http://structure.bmc.lu.se/PON-SC Operating system(s): Linux.

Programming language: Python.

Any restrictions to use by non-academics: contact authors.

\section{Additional file}

Additional file 1: Table S1. Results for SCWRL + PROBE on validation dataset. (PDF $13 \mathrm{~kb}$ )

\section{Abbreviations}

AAS: Amino acid substitution; BTK: Bruton tyrosine kinase; ELANE: Elastase, neutrophil expressed; FN: False negative; FP: False positive; KRAS: KRAS protooncogene, GTPase; MCC: Matthews correlation coefficient; NGS: Next generation sequencing; NPV: Negative predictive value; PIK3R1: Phosphoinositide-3-kinase regulatory subunit 1; PPV: Positive predictive value; RASA1: RAS p21 protein activator 1; SH2D1A: SH2 domain-containing 1A; SMAD4: SMAD family member 4; STAT1: Signal transducer and activator of transcription 1; TN: True negative; TNF: Tissue necrosis factor; TNR: True negative rate; TP: True positive; TP53: Tumor protein p53; TPR: True positive rate; ZAP70: Zeta chain of T cell receptor associated protein kinase 70

\section{Acknowledgements}

Financial support from Swedish Research Council, the Swedish Childhood Cancer Foundation and Alfred Österlund Foundation is gratefully acknowledged.

\section{Funding}

This work has been supported by the Swedish Research Council, the Swedish Childhood Cancer Foundation and Alfred Österlund Foundation.

\section{Availability of data and materials}

The datasets generated and/or analyzed during the current study are available in the VariBench repository, http://structure.bmc.lu.se/NariBench/sidechain.php

Authors' contributions

JČ programmed the predictor, tested the tools, analysed results and drafted the manuscript. MV conceived the idea, supervised the project, analysed results and drafted the manuscript. Both authors read and approved the final manuscript. 


\section{Ethics approval and consent to participate}

Not applicable.

\section{Consent for publication}

Not applicable.

\section{Competing interests}

The authors declare that they have no competing interests.

\section{Author details}

'Protein Structure and Bioinformatics, Department of Experimental Medical Science, Lund University, BMC B13, SE-22 184 Lund, Sweden. ${ }^{2}$ Present address: EMBL Heidelberg, Meyerhofstraße 1, 69117 Heidelberg, Germany.

Received: 14 June 2017 Accepted: 21 November 2017

Published online: 29 November 2017

\section{References}

1. Vihinen M. Types and effects of protein variations. Hum Genet. 2015;134: 405-21.

2. Niroula A, Urolagin S, Vihinen M. PON-P2: prediction method for fast and reliable identification of harmful variants. PLoS One. 2015;10(2):e0117380.

3. Kircher M, Witten DM, Jain P, O'Roak BJ, Cooper GM. A general framework for estimating the relative pathogenicity of human genetic variants. Nat Genet. 2014;46:310-5.

4. Schwarz JM, Cooper DN, Schuelke M, Seelow D. MutationTaster2: mutation prediction for the deep-sequencing age. Nat Methods. 2014;11:361-2.

5. Yang Y, Chen B, Tan G, Vihinen M, Shen B. Structure-based prediction of the effects of a missense variant on protein stability. Amino Acids. 2013;44:847-55.

6. Cheng J, Randall A, Baldi P. Prediction of protein stability changes for singlesite mutations using support vector machines. Proteins. 2006;62:1125-32

7. Yin S, Ding F, Dokholyan NV. Eris: an automated estimator of protein stability. Nat Methods. 2007:4:466-7.

8. Ali H, Urolagin S, Gurarslan O, Vihinen M. Performance of protein disorder prediction programs on amino acid substitutions. Hum Mutat. 2014;35:794-804.

9. Conchillo-Sole O, de Groot NS, Aviles FX, Vendrell J, Daura X, Ventura S. AGGRESCAN: A server for the prediction and evaluation of "hot spots" of aggregation in polypeptides. BMC bioinformatics. 2007;8:65.

10. Trovato A, Seno F, Tosatto SC. The PASTA server for protein aggregation prediction. Protein Eng Des Sel. 2007;20:521-3.

11. Laurila K, Vihinen M. PROlocalizer: integrated web service for protein subcellular localization prediction. Amino Acids. 2011:40:975-80.

12. Desmet FO, Hamroun D, Lalande M, Collod-Beroud G, Claustres M, Beroud C. Human Splicing Finder: an online bioinformatics tool to predict splicing signals. Nucleic Acids Res. 2009;37(9):e67.

13. Mort M, Sterne-Weiler T, Li B, Ball EV, Cooper DN, Radivojac P, Sanford JR, Mooney SD. MutPred splice: machine learning-based prediction of exonic variants that disrupt splicing. Genome Biol. 2014;15:R19.

14. Niroula A, Vihinen M. PON-mt-tRNA: a multifactorial probability-based method for classification of mitochondrial tRNA variations. Nucleic Acids Res. 2016:44:2020-7.

15. Kondrashov FA. Prediction of pathogenic mutations in mitochondrially encoded human tRNAs. Hum Mol Genet. 2005;14:2415-9.

16. Niroula A, Vihinen M. Variation interpretation predictors: principles, types, performance, and choice. Hum Mutat. 2016:37:579-97.

17. Thusberg J, Vihinen M. Pathogenic or not? And if so, then how? Studying the effects of missense mutations using bioinformatics methods. Hum Mutat. 2009;30:703-14.

18. Lindor NM, Guidugli L, Wang X, Vallee MP, Monteiro AN, Tavtigian S, Goldgar DE, Couch FJA. Review of a multifactorial probability-based mode for classification of BRCA1 and BRCA2 variants of uncertain significance (VUS). Hum Mutat. 2012;33:8-21.

19. Goldgar DE, Easton DF, Byrnes GB, Spurdle AB, Iversen ES, Greenblatt MS. Genetic evidence and integration of various data sources for classifying uncertain variants into a single model. Hum Mutat. 2008;29:1265-72.

20. Niroula A, Vihinen M. Classification of amino acid substitutions in mismatch repair proteins using PON-MMR2. Hum Mutat. 2015;36:1128-34.

21. Chao EC, Velasquez JL, Witherspoon MS, Rozek LS, Peel D, Ng P, Gruber SB, Watson P, Rennert G, Anton-Culver H, et al. Accurate classification of MLH1/ MSH2 missense variants with multivariate analysis of protein polymorphisms-mismatch repair (MAPP-MMR). Hum Mutat. 2008;29:852-60.
22. Väliaho J, Faisal I, Ortutay C, Smith CIE, Vihinen M. Characterization of all possible single nucleotide change-caused amino acid substitutions in the kinase domain of Bruton tyrosine kinase. Hum Mutat. 2015:36:638-47.

23. Niroula A, Vihinen M. Predicting severity of disease-causing variants. Hum Mutat. 2017:38:357-64.

24. Shapovalov MV, Dunbrack RL Jr. A smoothed backbone-dependent rotamer library for proteins derived from adaptive kernel density estimates and regressions. Structure (London, England). 2011;19:844-58.

25. Lovell SC, Word JM, Richardson JS, Richardson DC. The penultimate rotamer library. Proteins. 2000;40:389-408.

26. Towse CL, Rysavy SJ, Vulovic IM, Daggett V. New Dynamic Rotamer Libraries: Data-Driven Analysis of Side-Chain Conformational Propensities. Structure (London, England). 2016;24:187-99.

27. Krivov GG, Shapovalov MV, Dunbrack RL Jr. Improved prediction of protein side-chain conformations with SCWRL4. Proteins. 2009;77:778-95.

28. Nagata K, Randall A, Baldi P. SIDEpro: a novel machine learning approach for the fast and accurate prediction of side-chain conformations. Proteins. 2012;80:142-53.

29. Wright JD, Lim CA. Fast method for predicting amino acid mutations that lead to unfolding. Protein Eng. 2001:14:479-86.

30. Word JM, Bateman RC Jr, Presley BK, Lovell SC, Richardson DC. Exploring steric constraints on protein mutations using MAGE/PROBE. Protein Sci. 2000;9:2251-9.

31. Pottel J, Moitessier N. Single-point mutation with a Rotamer library toolkit: toward protein engineering. J Chem Inf Model. 2015;55:2657-71.

32. Rouet $R$, Lowe $D$, Christ $D$. Stability engineering of the human antibody repertoire. FEBS Lett. 2014:588:269-77.

33. Socha RD, Tokuriki N. Modulating protein stability - directed evolution strategies for improved protein function. FEBS J. 2013;280:5582-95.

34. O'Fagain C. Engineering protein stability. Methods in molecular biology (Clifton, NJ). 2011:681:103-36.

35. Poultney CS, Butterfoss GL, Gutwein MR, Drew K, Gresham D, Gunsalus KC, Shasha DE, Bonneau R. Rational design of temperaturesensitive alleles using computational structure prediction. PLoS One. 2011;6:e23947.

36. Tan KP, Khare S, Varadarajan R, Madhusudhan MS. TSpred: a web server for the rational design of temperature-sensitive mutants. Nucleic Acids Res. 2014:42:W277-84

37. Thusberg J, Vihinen M. The structural basis of hyper IgM deficiency - CD40L mutations. Protein Eng Des Sel. 2007;20:133-41.

38. Thusberg J, Vihinen M. Bioinformatic analysis of protein structure-function relationships: case study of leukocyte elastase (ELA2) missense mutations. Hum Mutat. 2006:27:1230-43.

39. Cock PJ, Antao T, Chang JT, Chapman BA, Cox CJ, Dalke A, Friedberg I, Hamelryck T, Kauff F, Wilczynski B, et al. Biopython: freely available python tools for computational molecular biology and bioinformatics. Bioinformatics. 2009;25:1422-3

40. Frishman D, Argos P. Knowledge-based protein secondary structure assignment. Proteins. 1995;23:566-79.

41. Pedregosa F, Varoquaux G, Gramfort A, Michel V, Thirion B, Grisel O, Blondel $M$, Prettenhofer $P$, Weiss R, Dubourg $V$, et al. Scikit-learn: machine learning in python. J Mach Learn Res. 2011:12:2825-30.

42. Hooft RW, Vriend G, Sander C, Abola EE. Errors in protein structures. Nature. 1996;381:272.

43. Jeffrey G. An introduction to hydrogen bonding. New York: Oxford University Press; 1997.

44. Richardson JS. The anatomy and taxonomy of protein structure. Adv Protein Chem. 1981:34:167-339.

45. Berman HM, Westbrook J, Feng Z, Gilliland G, Bhat TN, Weissig H, Shindyalov IN, Bourne PE. The Protein Data Bank. Nucleic Acids Res. 2000;28:235-42.

46. Nair PS, Vihinen M. VariBench: a benchmark database for variations. Hum Mutat. 2013;34:42-9

47. Word JM, Lovell SC, LaBean TH, Taylor HC, Zalis ME, Presley BK, Richardson JS, Richardson DC. Visualizing and quantifying molecular goodness-of-fit: small-probe contact dots with explicit hydrogen atoms. J Mol Biol. 1999;285:1711-33.

48. Lappalainen I, Thusberg J, Shen B, Vihinen M. Genome wide analysis of pathogenic SH2 domain mutations. Proteins. 2008;72:779-92.

49. Shi Z, Moult J. Structural and functional impact of cancer-related missense somatic mutations. J Mol Biol. 2011;413:495-512. 
50. Vihinen M. How to evaluate performance of prediction methods? Measures and their interpretation in variation effect analysis. BMC genomics. 2012; 13(Suppl 4):S2

51. Vihinen M. Guidelines for reporting and using prediction tools for genetic variation analysis. Hum Mutat. 2013;34:275-82.

52. Eyal E, Najmanovich R, Edelman M, Sobolev V. Protein side-chain rearrangement in regions of point mutations. Proteins. 2003;50:272-82.

Submit your next manuscript to BioMed Central and we will help you at every step:

- We accept pre-submission inquiries

- Our selector tool helps you to find the most relevant journal

- We provide round the clock customer support

- Convenient online submission

- Thorough peer review

- Inclusion in PubMed and all major indexing services

- Maximum visibility for your research

Submit your manuscript at www.biomedcentral.com/submit 